\title{
QUANTUM TRANSPORT SIMULATIONS OF ELECTRON FIELD EMISSION
}

\author{
K. L. Jensen and A. K. Ganguly
}

Electronics Science and Technology Division

Naval Research Laboratory, Washington, DC 20375-5000

\begin{abstract}
We report on the comparison of various quantum transport calculations for metal - vacuum tunneling structures under high electric fields. Specifically, we shall compare the WKB approximation to the Transmission Coefficient (TC) for evaluating current - voltage relationships to the more accurate Airy function approach, and both of these approaches to the Wigner Distribution Function (WDF) approach to quantum transport. The purpose of this study is to examine electron transport through metal (or semiconductor) - vacuum interfaces and to assess the utility of various techniques for their incorporation into larger simulation packages, such as Ensemble Particle Monte Carlo. As the WDF has been used for the first time to study issues of interest to Vacuum Microelectronics (VM), we assess its advantages and shortcomings.
\end{abstract}

\section{INTRODUCTION}

It is desirable to construct a numerical simulation approach to quantum transport in order to examine fundamental issues of electron emission from the surface of a metal or semiconductor into the vacuum, which necessarily should include: open boundary conditions to allow particles to enter and exit the simulation region; incorporation of time-dependent phenomena; electron-electron and other phenomenological interactions; and finally, as the entire tunneling process takes place in a region smaller than $100 \AA$, the simulation package should eventually be able to couple to EPMC approaches which are the work-horses of general simulations in order to model devices whose size dwarfs the tunneling region.

TC calculations [1] provide a fairly robust method for extracting current - voltage relationships for relatively low fields $(0.1$ to $0.5 \mathrm{~V} / \AA)$. If computer time, and in some cases, memory, are not an issue, many body effects and scattering (to an extent) can be dealt with [2]. To our knowledge, though, an "open," self-consistent, time-dependent, many body treatment has not yet emerged. Since our intention is to examine and compare the relatively fast methods, we shall deal with the simplest (from a numerical point of view) of the TC calculations, especially since they form the basis of much of the theoretical work done in VM, in part because many-body and scattering effects are not deemed to be as important [3] in VM as they are in, for example, semiconductor simulations.

A brief list of the advantages of the WDF approach [3] are: modeling of open systems; scattering and many-body effects are easily incorporated; the phase space language provides a means for coupling quantum transport simulations to EPMC simulations via "quantum particle trajectories" [5]; whereas simple TC calculations neglect the interaction of the plane wave phases, these interactions are included in the WDF implicitly; finally, as self-consistency and scattering are trivial modifications to its defining matrix equation, the WDF approach is fast and uncomplicated to impliment (this alone would make it quite attractive compared to TC). However, in spite of successes in examining quantum well devices, extracting current - voltage relationships for low electric fields has proven difficult for typical metal-vacuum parameters for the WDF.

\section{THE TRANSMISSION COEFFICIENT}

The ubiquitous Fowler-Nordheim (FN) equation may be calculated from the equation for current:

$$
\mathrm{J}_{\mathrm{TC}}=\frac{1}{2 \pi} \int_{0}^{\infty} \mathrm{dk} \frac{\hbar \mathrm{k}}{\mathrm{m}} \mathrm{f}_{\mathrm{o}}(\mathrm{k}) \mathrm{T}(\mathrm{k})
$$

(where $\mathrm{f}_{\mathrm{o}}(\mathrm{k}$ ) is the one-dimensional Fermi-Dirac distribution, $(\mathrm{hk} / \mathrm{m})$ is the velocity, and $T(k)$ is the Transmission coefficient as a function of field) by making the following approximations: (a) the majority of the current comes from a small region around the Fermi energy; (b) the WKB approximation is valid for the transmission coefficient; (c) as a result of (a) and (b), the log of the transmission coefficient may be assumed to be linear in energy in the region near the Fermi energy; (d) an image charge potential is included; and finally (e) the zero temperature limit is taken. As a result of these approximations, the FN equation is independent of both temperature and chemical potential .

The WKB approach makes approximations in turn [6]. Given the widespread use of both the FN equation and the WKB approach $[1,3$ and references therein] in the treatment of fundamental issues of VM, it is of considerable interest to see how these methods fare against a more rigorous evaluation of TC. The method we have used to evaluate $T(K)$ is to assume the potential is piecewise linear and then to match the wave function and its derivatives at the points where the slope in the potential changes [8]. In Figure 1, we compare the results of the calculation. In Figure 2, the percent deviation of the WKB and FN approximations is typically between $20 \%$ and $30 \%$. Note that this rather fortuitous agreement is sensitive to the value of the Fermi level; if the chemical potential were to decrease from $5.0 \mathrm{eV}$ to $1.0 \mathrm{eV}$, the \% error for the WKB approach would not drop below $40 \%$. Figure 3 gives a hint as to why this happens: in the vicinity of the Fermi level, the slope of the log of TC for WKB and the exact solution differ; the exponential of a small change can lead to large differences.

\section{THE WIGNER FUNCTION}

The WDF is a phase space description of quantum mechanics. The motion of the phase space points serve to define by "quantum particle trajectories." Note that since the Wigner function is not a true probability distribution function, the motion of the phase space points do not correspond to the

\subsection{1}

U.S. Government work not protected by U.S. copyright. 
motion of actual particles, but the behavior of these trajectories is so suggestive as to argue for their usage in situations where a trajectory approach is demanded (such as in EPMC). The outline of a possible method for accomplishing this has been given before $[5,7]$.

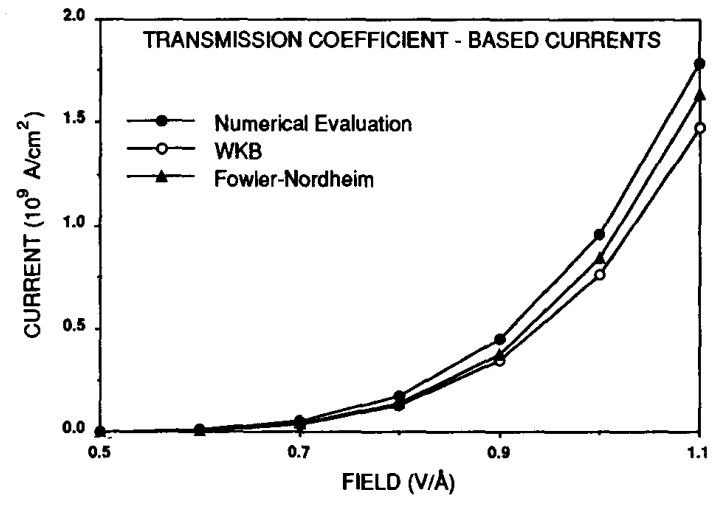

Figure 1: Current-Voltage characteristics for a typical metal-vacuum potential of electric field + image charge. The work function was taken to be $4.35 \mathrm{eV}$, and the chemical potential $5.0 \mathrm{eV}$. The temperature was $300 \mathrm{~K}$.

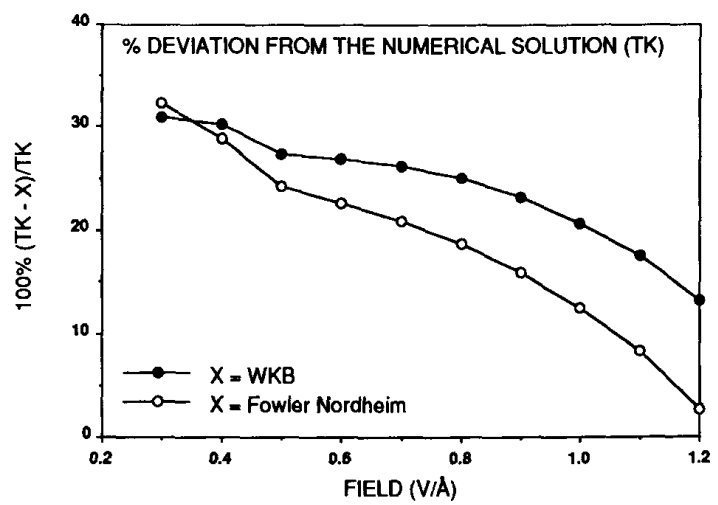

Figure 2: \% deviation of current estimates of the approximations from the actual (Airy) solution. For fields of interest, the deviation does not drop below $20 \%$.

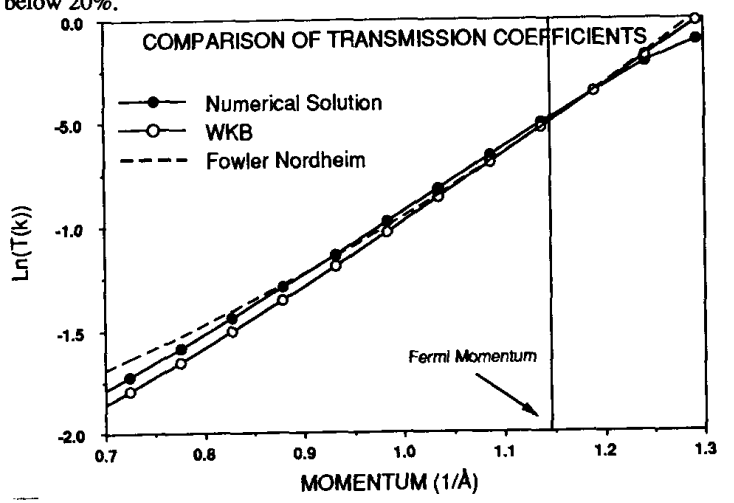

Figure 3: Logarithm of the transmission coefficient. Small deviations in the slope compared to the numerical value near the Fermi level give rise to significant differences in the current.
The advantages of WDF should not detract from the fact that it is at present a Faustian bargain for VM since, at present we have not been able to extract reasonable currents from the WDF simulations for typical metal-vacuum parameters. It is anticipated that this is but a temporary setback; therefore, below, we shall use parameters for which WDF does give meaningful results. First, however, we shall briefly discuss what we believe are the difficulties with the WDF approach [10].

\section{i) Discretiztion:}

Due to the magnitude of the chemical potential for metals, the distribution of incoming electrons has a relatively sharp (from a numerical standpoint) discontinuity at the Fermi momentum. Consequently, k-space should be relatively well-resolved. The magnitude and sharpness of the potential term $\mathrm{V}(\mathrm{x})$ also demands that position space be well-resolved. However, an increased resolution in $x$-space cannot accompany increased resolution in $\mathbf{k}$-space unless one is willing to forgo defining the potential on the same lattice that the WDF is defined upon [7], or unless a larger number of $\mathrm{x}$ - and $\mathrm{k}$-space points are included.

\section{ii) Potential Profile:}

The metal-vacuum interface is not only sharp, but the abrupt portions of the potential are non-symmetrical. The discretized solution of the Wigner function works best if the sharp discontinuities in the potential are symmetrically placed about the center of the simulation region; if not, unphysical currents that scale with the discretization spacing are introduced (see Frensley). There are two methods to deal with this difficulty: (a) simulate a metal-vacuum-metal system so that the symmetry is restored, or (b) move to finer and finer discretizations in $\mathrm{x}$-space. The former approach is a bit removed from the micron or so spacings between contacts that are inherent to FEA's, in spite of its beneficial effects on the stability of the matrix solution. Increasing the number of mesh points never really eliminates the spurious currents but only suppresses them in a manner that becomes more time- and memory-intensive as $\Delta x$ and $\Delta k$ become smaller.

\section{iii.) Truncation in momentum space:}

Due to the sharpness of the barrier, the WDF extends greatly in momentum space near the metal-vacuum interface. If the phase space is truncated at too small a value of momentum the errors introduced propagate back into the low momentum regions of phase space, causing non-physical ripples and oscillations. When evaluating the density, these errors are not too severe, but when evaluating the current, which relies on fine differences between the positive and negative momentum regions of the WDF, the problems are much more acute. If one extends $\mathrm{k}$-space to avoid this problem, errors associated with $\Delta \mathrm{k}$ being too coarse are introduced.

\section{COMPARISON OF TC AND WDF FOR A SIMPLE RECTANGULAR BARRIER}

For purposes of comparison, let us take a model system defined by the following: imagine that two slabs of metal are separated by a small region of vacuum; further, assume that the electric field is constant between the two metal plates (i.e., the potential is linear), as in Figure 4. Let the height of the barrier be given by $1.6 \mathrm{eV}$, and the separation by $10 \mathrm{~A}$. Even though this potential does not vary smoothly, it is still possible to show that the TC has a form analogous to the WKB approximation [6], and a short calculation shows:

$$
\left.T(k)=\exp \left\{-\frac{4}{3 f}\left[\left(v-k^{2}\right)^{3 / 2}-\left(v-f w-k^{2}\right)^{3 / 2}\right)\right]\right\}
$$

where $v=2 m \phi / \hbar^{2}, f=2 m F / \hbar^{2}$, and $w=$ width of the barrier, 
and it is assumed that $(v-f w)$ is greater than $k^{2}$ (the second term in the exponential is not present when $\left.k^{2}>v-w f\right)$.

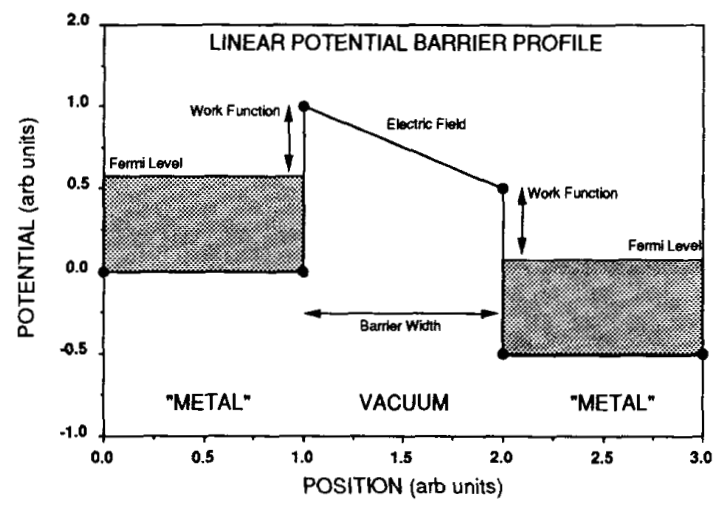

Figure 4: Potential profile used to compare transmission coefficient to Wigner function evaluations of the current. Note that electrons will be striking the potential barrier from both sides: left (or upwind) and right (or downwind).

In Figure 5, we show a comparison of the currents evaluated by the approximate form of TC, the Airy form of TC, and the WDF approach, where particles are assumed to be incident on both sides of the barrier. The parameters for the calculations were: chemical potential $\mu=0.6 \mathrm{eV}$, barrier height $=1.0 \mathrm{eV}$, and temperature $=300 \mathrm{~K}$. For the $\mathrm{WDF}, \mathrm{Nk}=128$, $\mathrm{Nx}=160, \Delta \mathrm{x}=0.5 \AA$ (for the $10 \AA$ barrier) and $0.6 \AA$ (for the $6 \AA$ barrier $), \Delta \mathrm{k}=\Delta \mathrm{x} /(\pi \mathrm{Nk})$.

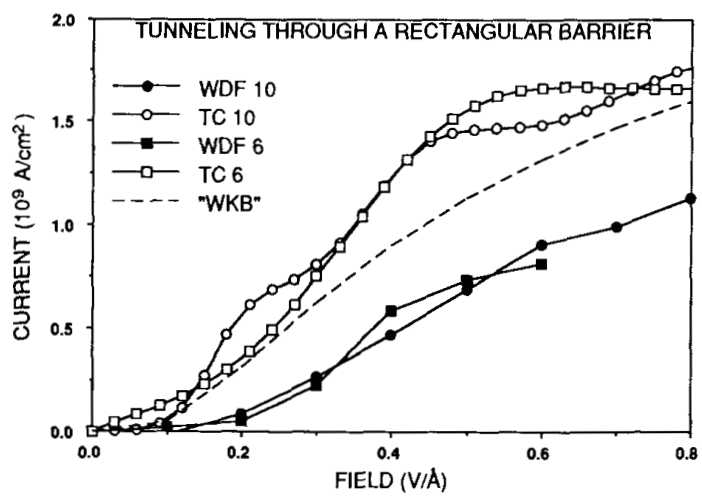

Figure 5: Comparison between transmission coefficient and Wigner function evaluations of current for the barrier shown in Figure 4 . The chemical potential was taken to be $0.6 \mathrm{eV}$, the work function $1.0 \mathrm{eV}$. " 6 " and "10" refer to the barrier width in angstroms. "WKB" refers to the approximation formula given in Equation 2.

A few comments are in order: (a) the current is due to particles incident from the left side of the barrier for all the approaches for fields greater than $0.1 \mathrm{~V} / \AA \AA$; (b) the "ripples" in the curves for both TC and WDF are due to the presence of the second downstream metal-vacuum interface - when this interface is "infinitely" far away (the typical Fowler-Nordheim scenario), these ripples are smoothed out, much like the WKB curve; (c) the current estimated by the WDF is consistently smaller than that estimated by the TC approach; (d) the appearance of the TC and WDF curves appear qualitatively similar, but note that the ripples, due to interference effects, are a bit different. That (c) occurs may be considered surprising: it is possible that some of the difference is because the discrete Fourier transform is performed on the potential; consequently, depending on the $k$-space discretization, the potential barriers may appear to be larger at the metal-vacuum interfaces than they actually are [9]; but even so, this would only comprise a small part of the difference. A larger part is undoubtedly due to the differences in the physics incorporated by the two methods; for instance, the Wigner function $f(x, k)$ is "approximated" by $f_{o}(k) T(k)$ for the transmission coefficient approach in the current integrand.

\section{WDF'S AND TRAJECTORIES FOR METAL TO VACUUM TUNNELING}

Finally, we turn our attention to WDF simulations of a simple metal-vacuum interface. The parameters for the calculation were: chemical potential $\mu=0.5 \mathrm{eV}$, work function $\phi=3.1 \mathrm{eV}$, temperature $=300 \mathrm{~K}, \mathrm{Nk}=160, \mathrm{Nx}=180, \Delta \mathrm{x}=$ $0.2235 \AA, \Delta \mathrm{k}=0.027341 / \AA$, metal-vacuum boundary located at $28.0 \AA$, potential profile $=\phi+\mu-\mathrm{eEx}-\mathrm{e}^{2} / 2 \mathrm{x}$. In Figure 6 , we show the results of one such calculation for an applied field of $0.6 \mathrm{~V} / \AA$. In form, the WDF is very similar to the upwind sections of the WDF's calculated previously. The trajectories for this WDF are shown in Figure 7. For steady state calculations, these trajectories are synonymous with the contour lines of the WDF. That these trajectories may be suitable for use in a Monte Carlo calculation is indicated by the fact that near $x=$ $40 \AA$, the "force" acting on the largest tunneling trajectory is close in magnitude to the classical value of $-\mathrm{eE}+\mathrm{e}^{2} / 2 \mathrm{x}^{2}[10]$. Consequently, the Quantum forces appear to merge naturally into their classical counterparts "far" from the tunneling region ("far" can be taken to mean $15 \AA$ or so). It remains to be seen whether the trajectory approach can be used to estimate current through a barrier by summing over trajectories and their respective weights [11] for potentials associated with VM.

\section{WIGNER DISTRIBUTION FUNCTION FOR FIELD EMISSION}

Applied Electric Fletd Of $0.6 \mathrm{~V} / \mathrm{A}$

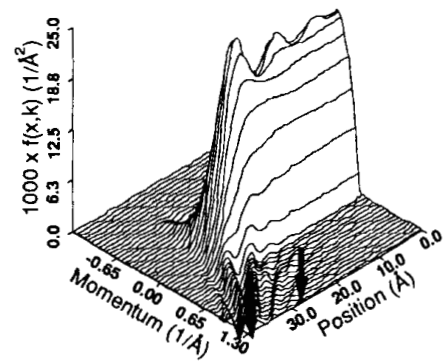

Figure 6: The Wigner function for a metal vacuum potential of electric field + image charge. The work function was $3.1 \mathrm{eV}$, the chemical potential $0.5 \mathrm{eV}$, and the field $0.6 \mathrm{~V} / \AA$. The arrow indicates the location of the metal vacuum interface.

The incorporation of the "trajectory" concept into an EPMC program may be done by invoking the "Quantum Force" in the vicinity of the tunneling region for calculating the forces acting on the EPMC particles [5,7]. Used in this way, the Quantum Force calculated from the WDF is formally very similar to the Quantum Potential calculations performed by $D$. Bohm and others in their investigations of wave packet tunneling phenomena [11]. Further, Wigner trajectories have been fruitfully employed for resonant tunneling diode simulations 
'[4,5,7] and elsewhere [13]; perhaps a similar state of affairs will occur for the treatment of field emission after the resolution of the difficulties outline above.

\section{STEADY STATE TRAJECTORIES}

Appled Electric Field of 0.6 V/A

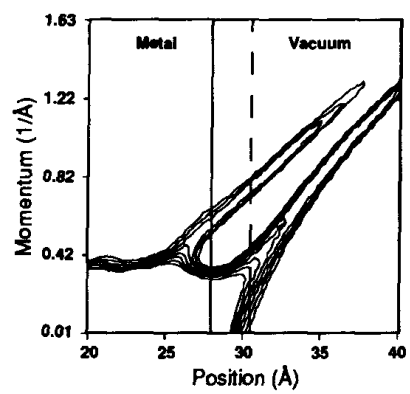

Figure 7: The trajectories associated with the Wigner function shown in Figure 6.

\section{CONCLUSION}

In this work, we have compared two methods for evaluating current through various one dimensional potential profiles, first, by numerically solving Schrodinger's equation (via a piece-wise Airy function approach) to extract the "exact" transmission coefficient, and second, by solving for the Wigner distribution function. The TC calculations were first compared to the Fowler Nordheim equation, and then to the WKB approximation for the field emission problem to assess the accuracy of the approximations. Disagreement by $20 \%$ or more in the range of interest was found. Next, the TC calculations were compared with the WDF approach to tunneling through a simple rectangular barrier, and was found to overestimate the current by comparison to the WDF current. For the WDF, issues concerning its numerical evaluation were discussed. Last, the WDF was evaluated for the field emission problem, and from it, the "particle trajectories" were constructed. Far from the tunneling region, the forces acting on the trajectories are comparable to the classical values, which bolsters the claim that trajectories may be useful for incorporating quantum tunneling into a Monte Carlo simulation of electron emission by use of a "quantum force" calculated from the Wigner function.

\section{ACKNOWLEDGEMENT}

We would like to thank the Office of Naval Research and the Office of Naval Technology for supporting this project. KLJ would like to thank E. G. Zaidman and F. A. Buot for numerous discussions.

\section{REFERENCES}

[1] P. H Cutler T. E. Feuchtwang, Z. Huang, and T. Sullivan, Vacuum Microelectronics 1989, Inst. Conf. Ser. 99 (IOP, London, 1989); Modinos, A., Field, Thermionic, and Secondary Electron Emission Spectroscopy, (Plenum Press, New York, 1984).

[2] See, for example, M. Cahay, M. McLennan, S. Datta, and M. S. Lundstrom, Applied Physics Letters 50, 612, (1987), and references therein

[3] P. H. Cutler, Jun He, N. M. Miskovsky, T. E Feuchtwang, and T. E. Sullivan, "Theory of Electron Emission in High Fields from Atomically Sharp Emitters," Technical Digest of the Fourth International Vacuum Microelectronics Conference, Business Center for Academic Societies Japan (BCASJ), Osaka, Japan (Aug. 22-24, 1991); P. H. Cutler (private communication).

[4] K. L. Jensen and F. A. Buot, Physical Review Letters 66 1078 (1991), Physical Review B 42, 9429 (1990); W. R. Frensley, Reviews of Modern Physics 62, 745 (1990); J. Barker, Physica 134B, 22 (1985); H. Tsuchiya, M. Ogawa, and T. Miyoshi, IEEE Transctions on Electron Devices 38, 1246 (1991).

[5] K. L. Jensen and F. A. Buot, "The Numerical Simulation of Particle Trajectories in Quantum Transport and the Effects of Scattering and Self-consistency on the Performance of Quantum Well Devices," Technical Digest of the IEEE IEDM, sec. 31.4 (1990).

[6] S. Gasiorowicz, Quantum Physics, New York: Wiley, 1974; E. Merzbacher, Quantum Mechanics, 2nd Ed., New York: Wiley, 1970.

[7] K. L. Jensen and F. A. Buot, IEEE Transctions on Electron Devices 38, 2337 (1991)

[8] Our method is similar to, but not identical, to: K. F. Brennan and C. J. Summers, Journal of Applied Physics 61, 614 (1987); C. M. Tan, J. Xu, and S. Zukotynski, Journal of Applied Physics 67, 3011 (1990); H. Inaba, K. Kurosawa, and M. Okuda, Japanese Journal of Applied Physics 28, 2201 (1989).

[9] See, for example, the treatment of the Fourier Series for square and sawtooth functions in: G. Arfken, Mathematical Methods for Physicists, 3rd Ed, New York: Academic Press, 1985; E. O. Brigham, The Fast Fourier Transform, Englewood Cliffs, NJ: Prentice-Hall, 1974.

[10] K L. Jensen and A. K. Ganguly, "The Numerical Simulation of Electron Emission from Field Emitters Using the Wigner Distribution Function Approach," Technical Digest of the Fourth International Vacuum Microelectronics Conference, Business Center for Academic Societies Japan (BCASJ), Osaka, Japan (Aug. 22-24, 1991).

[11] Some calculations which strongly suggest that this may in fact occur can be found in: $K$. L. Jensen and F. A. Buot, Applied Physics Letters, 55669 (1989).

[12] D. Bohm and B. J. Hiley, Nuovo Cimento, 52, 246 (1979).; C. Dewdney and B. J. Hiley, Foundations of Physics, 12, 27 (1982).

[13] H-W. Lee and M. O. Scully, Foundations of Physics 13, 61 (1983) and references therein. 